\title{
Selenium uptake, toxicity and reduction in Flammulina velutipes supplied with selenite
}

Jipeng Wang, Bo Wang, Dan Zhang, Yanhong Wu

Recently, selenium (Se) enriched mushrooms have been exploited as diary Se supplements, but our knowledge of the metabolism process during the Se enrichment process is far from complete. In this study, the uptake, toxicity and reduction of selenite in a widely cultivated mushroom, Flammulina velutipes, was investigated. The results showed that $\mathrm{pH}$ variation (from 5.5 to 7.5), metabolic inhibitor (0.1 mM 2,4-DNP) and $\mathrm{P}$ or $\mathrm{S}$ starvation led to $11 \%-26 \%$ decreases in the selenite uptake rate of $F$. velutipes. This indicates that a minor portion of the selenite uptake was metabolism dependent, whereas a carrier-facilitated passive transport may be crucial. Growth inhibition of $F$. velutipes initiated at $0.1 \mathrm{mM}$ selenite ( $11 \%$ decrease in the growth rate) and complete growth inhibition occurred at $3 \mathrm{mM}$ selenite. A selenite concentration of 0.03-0.1 mM was recommended to maintain the balance between mycelium production and Se enrichment.

$F$. velutipes was capable of reducing selenite to $\mathrm{Se}(0)$ nanoparticles, possibly as a detoxification mechanism. This process depended on both selenite concentration and metabolism activity. Overall, the data obtained provided some basic information for the cultivation of the selenized $F$. velutipes, and highlighted the opportunity of using mushrooms for the production of $\mathrm{Se}(0)$ nanoparticles. 


\section{Selenium uptake, tolerance and reduction in Flammulina velutipes}

\section{2 supplied with selenite}

3 Jipeng Wang ${ }^{1,2}$, Bo Wang ${ }^{3}$, Dan Zhang ${ }^{1}$, Yanhong $\mathrm{Wu}^{1}$

$4{ }^{1}$ Key Laboratory of Mountain Surface Process and Ecological Regulation, Institute of Mountain Hazards and

5 Environment, Chinese Academy of Sciences (CAS), Chengdu, China

6 '2University of Chinese Academy of Sciences, CAS, Beijing, China

$7 \quad{ }^{3}$ Soil and Fertilizer Institute, Sichuan Academy of Agricultural Sciences, Chengdu, China

$8 \quad$ Yanhong $\mathrm{Wu}$ (corresponding author)

$9 \quad \# 9$, Block 4, Renminnanlu Road, Chengdu, Sichuan, 610041, China.

E-mail address: yhwu@imde.ac.cn

Abstract: Recently, selenium (Se) enriched mushrooms have been exploited as diary Se supplements, but our knowledge of the metabolism process during the Se enrichment process is far from complete. In this study, the uptake, tolerance and reduction of selenite in a widely cultivated mushroom, Flammulina velutipes, was investigated. The results showed that $\mathrm{pH}$ variation (from 5.5 to 7.5 ), metabolic inhibitor ( $0.1 \mathrm{mM} 2,4-\mathrm{DNP}$ ) and $\mathrm{P}$ or S starvation led to $11 \%$ $26 \%$ decreases in the selenite uptake rate of $F$. velutipes. This indicates that a minor portion of the selenite uptake was metabolism dependent, whereas a carrier-facilitated passive transport may be crucial. Growth inhibition of $F$. velutipes initiated at $0.1 \mathrm{mM}$ selenite $(11 \%$ decrease in the growth rate) and complete growth inhibition occurred at $3 \mathrm{mM}$ selenite. A selenite concentration of $0.03-0.1 \mathrm{mM}$ was recommended to maintain the balance between mycelium production and Se enrichment. F. velutipes was capable of reducing selenite to elemental Se $[\mathrm{Se}(0)]$ including $\mathrm{Se}(0)$ nanoparticles, possibly as a detoxification mechanism. This process depended on both selenite concentration and metabolism activity. Overall, the data obtained provided some basic information for the cultivation of the selenized $F$. velutipes, and highlighted the opportunity of using mushrooms for the production of $\operatorname{Se}(0)$ nanoparticles. 


\section{Selenium uptake, tolerance and reduction in Flammulina velutipes}

\section{7 supplied with selenite}

28 Abstract: Recently, selenium (Se) enriched mushrooms have been exploited as diary Se

29 supplements, but our knowledge of the metabolism process during the Se enrichment process is

30 far from complete. In this study, the uptake, tolerance and reduction of selenite in a widely

31 cultivated mushroom, Flammulina velutipes, was investigated. The results showed that $\mathrm{pH}$

32 variation (from 5.5 to 7.5 ), metabolic inhibitor ( $0.1 \mathrm{mM}$ 2,4-DNP) and $\mathrm{P}$ or $\mathrm{S}$ starvation led to $11 \%$ -

$3326 \%$ decreases in the selenite uptake rate of $F$. velutipes. This indicates that a minor portion of

34 the selenite uptake was metabolism dependent, whereas a carrier-facilitated passive transport

35 may be crucial. Growth inhibition of $F$. velutipes initiated at $0.1 \mathrm{mM}$ selenite $(11 \%$ decrease in

36 the growth rate) and complete growth inhibition occurred at $3 \mathrm{mM}$ selenite. A selenite

37 concentration of $0.03-0.1 \mathrm{mM}$ was recommended to maintain the balance between mycelium

38 production and Se enrichment. F. velutipes was capable of reducing selenite to elemental Se

$39[\mathrm{Se}(0)]$ including $\mathrm{Se}(0)$ nanoparticles, possibly as a detoxification mechanism. This process

40 depended on both selenite concentration and metabolism activity. Overall, the data obtained

41 provided some basic information for the cultivation of the selenized $F$. velutipes, and highlighted

42 the opportunity of using mushrooms for the production of $\mathrm{Se}(0)$ nanoparticles. 


\section{Introduction}

44 Selenium (Se) is an essential trace element for humans and animals. Supplementation of Se can

45 be necessary, because nutritional Se deficiency affects 500-1000 million people worldwide,

46 especially those from the Keshan disease area of China (Combs 2001). The availability and

47 biological activity of Se depend on its dose and chemical form (Turło et al. 2011). In trace

48 amount, Se confers antioxidant capacities to a number of selenoproteins (Izquierdo et al. 2010).

49 At higher concentrations, $\mathrm{Se}$ is toxic because it generates oxidative stress and is involved in DNA damage (Izquierdo et al. 2010; Mániková et al. 2010). Organic Se-containing compounds

may exhibit anticarcinogenic activity, and their bioavailability to human beings and animals are considered to be high. (Chun et al. 2006; Suhajda et al. 2000). Recently, there has been increasing interest in using selenized mushrooms as a source of Se supplementation (EgressyMolnár et al. 2016; Maseko et al. 2013; Rzymski et al. 2016). Mushrooms have long been appreciated for their flavor and texture, as a nutritious food, and as a source of biologically active compounds (Cheung et al. 2003; Yan \& Chang 2012). Although the majority of mushrooms are Se-poor, a considerable amount of organic Se can accumulate in mushrooms supplied with selenite or other forms of Se (Falandysz 2008). Turło et al. (2011) reported that the mycelium of Lentinula edodes cultured in selenite-fortified substrate accumulated Se in organic compounds, and the synergetic effect of Se compounds and active polysaccharides gave it a strong chemopreventive activity. (Gharieb \& Gadd 2004), whereas the mechanism of selenite uptake by mushrooms remains 
64

65

unclear. Selenite is present in the culture medium in different forms depending on the $\mathrm{pH}$, including $\mathrm{H}_{2} \mathrm{SeO}_{3}, \mathrm{HSeO}_{3}{ }^{-}$and $\mathrm{SeO}_{3}{ }^{2-}$, which are mediated by different transporters. In algae, both specific and nonspecific transport systems are involved in the selenite uptake process (Araie et al. 2011; Morlon et al. 2006; Obata et al. 2004). The specific one is driven by $\Delta \mathrm{pH}$ energized by $\mathrm{H}^{+}$-ATPase (Araie et al. 2011), and both systems can be associated with transporters used by macronutrients such as phosphate, sulfate and nitrate (Araie et al. 2011; Morlon et al. 2006). In higher plants, selenite in the form of $\mathrm{H}_{2} \mathrm{SeO}_{3}$ is passively transported through aquaporins (Zhang et al. 2006; Zhang et al. 2010; Zhao et al. 2010), while $\mathrm{HSeO}_{3}{ }^{-}$is absorbed in a carrier-mediated way, sharing common transporters with phosphate or sulfite (Li et al. 2008; Zhang et al. 2006). In yeasts, selenite is found to be absorbed in a metabolism dependent way, using the transporter of phosphate or monocarboxylate (Gharieb \& Gadd 2004; Lazard et al. 2010; McDermott et al. 2010). Moreover, Gharieb \& Gadd (2004) also reported a fast, metabolism-independent process during the selenite uptake of Saccharomyces cerevisiae. Mushrooms may share similar pathways of selenite uptake with these eukaryotic organisms, and thus medium $\mathrm{pH}$, metabolism activity and competitive nutrients may serve as the major factors regulating the selenite uptake in mushrooms.

Mycelial growth and fructification of mushrooms can be hampered by toxic amounts of selenite in the growth substrate (Dong et al. 2012; Niedzielski et al. 2015; Nunes et al. 2012; Turło et al. 2010b). Therefore, an understanding of the tolerance of mushrooms to selenite is necessary to maintain the balance between mycelial production and Se enrichment. In addition to the growth changes when exposed to selenite, the mycelium of some mushrooms were reported 
to turn red due to the formation of $\operatorname{Se}(0)$ (Gharieb et al. 1995; Turło et al. 2010a; Vetchinkina et al. 2013; Vetchinkina et al. 2016). Bioreduction of selenite to $\operatorname{Se}(0)$ has long been observed (Levine 1925). Some bacteria and archaea grow anaerobically by linking the oxidation of organic substrates $\mathrm{H}_{2}$ to the dissimilatory reduction of Se oxyanions (Lovley 1993; Stolz \& Oremland 1999). Other organisms, including bacteria (Oremland et al. 2004), fungi (Falcone \& Nickerson 1963; Gharieb et al. 1995; Nickerson \& Falcone 1963) and plants (Zhu et al. 2009), can also reduce selenite to $\operatorname{Se}(0)$, most likely as a detoxification mechanism. The biologically formed Se(0) may partly exist as nanoparticles (Vetchinkina et al. 2013; Vetchinkina et al. 2016) which have novel biological activities (e.g., serve as an antioxidant, chemopreventive, and chemotherapeutic agent) and low toxicity (Zhang et al. 2012). Turło et al. (2010) suggested that $\mathrm{Se}(0)$, most likely in the nano-colloidal form, was responsible for the enhanced antioxidative activity of the mycelial extracts of the selenized $L$. edodes. Therefore, it is worthwhile to test mushrooms for the potentials of $\operatorname{Se}(0)$ production and to further examine the factors influencing this process.

Flammulina velutipes is among the four most widely cultivated mushrooms worldwide due to its desirable taste and nutritional values (Jing et al. 2014; Yang et al. 2016). With the high efficiency of Se accumulation (Lin et al. 1997), F. velutipes serves as a potential source of Se supplementation and biotransformation. In this study, we examined the uptake, tolerance and reduction of Se in $F$. velutipes supplied with $0-5 \mathrm{mM}$ of selenite. In addition, the abilities of $F$. velutipes in selenite tolerance and reduction were compared with 11 other species of mushrooms. Thus, the relative sensitivity of $F$. velutipes to selenite and the universality of the selenite 
106

107

108

109

110

111

112

113

114

115

116

117

118

119

120

121

122

123

124

125

126

reduction process among mushroom species can be understood. The objectives of this study were:

(1) to reveal the mechanisms of selenite uptake by F. velutipes; (2) to find out the optimal

concentration of selenite for the cultivation of selenized $F$. velutipes; (3) to test the ability of $F$.

velutipes to transform selenite to $\operatorname{Se}(0)$ and find out its influencing factors.

\section{Materials \& Methods}

\section{Strains and culture conditions}

The F. velutipes strain used in this study was obtained from the Soil and Fertilizer Institute,

Sichuan Academy of Agricultural Sciences, China. Stock cultures were maintained on glucoseyeast $(\mathrm{GY})$ agar plate consisting of glucose $(20 \mathrm{~g} / \mathrm{L})$, yeast extract $(5 \mathrm{~g} / \mathrm{L})$ and agar $(18 \mathrm{~g} / \mathrm{L})$ at $4^{\circ} \mathrm{C}$ in dark. Inoculum of $7 \mathrm{~mm}$ in diameter was picked up from the stock culture and inoculated onto the center of a GY agar plate ( $90 \mathrm{~mm}$ in diameter). After cultivated at $25^{\circ} \mathrm{C}$ in dark until $2 / 3$ of the plate was covered by fungal colony, the marginal parts of the fungal colony were used as sources of inocula for selenite uptake, tolerance and reduction experiments. For the other 11 species of mushrooms (Table S1 and S2), the same culture conditions were used as $F$. velutipes.

\section{Selenite uptake experiments}

Mycelial pellets for the uptake experiments were obtained through shaking cultivation in the GY medium for 12 days ( 2 inocula of $7 \mathrm{~mm}$ in diameter). The composition of the nutrient solution used for selenite uptake (uptake solution) was $15 \mathrm{~g} / \mathrm{L}$ glucose, $3 \mathrm{~g} / \mathrm{L}$ arginine, $1.37 \mathrm{~g} / \mathrm{L} \mathrm{KCl,} 0.5$ $\mathrm{g} / \mathrm{L} \mathrm{MgCl}_{2} \cdot 6 \mathrm{H}_{2} \mathrm{O}$. The $\mathrm{pH}$ of the solution was buffered at 6.0 with $2 \mathrm{mM}$ MES (2morpholinoethanesulphonic acid, $\mathrm{pH}$ adjusted with $\mathrm{NaOH}$ ). After the uptake process, a desorption solution was used to get rid of the selenite adhering on the surface of the mycelial 
127 pellets. The composition of the desorption solution was $0.136 \mathrm{~g} / \mathrm{L} \quad \mathrm{KH}_{2} \mathrm{PO}_{4}, 0.172 \mathrm{~g} / \mathrm{L}$

$128 \mathrm{CaSO}_{4} \cdot 2 \mathrm{H}_{2} \mathrm{O}$. The solution was buffered at $\mathrm{pH} 6.0$ with $2 \mathrm{mM} \mathrm{MES}$ and stored at $4{ }^{\circ} \mathrm{C}$. To test

129 the influence of $\mathrm{pH}$ on selenite uptake, the $\mathrm{pH}$ of the uptake solution was buffered at 5.5 and 6.5

130 with $2 \mathrm{mM}$ MES, and at 7.5 with 2 mM HEPES (4-(2-hydroxyethyl)-1-piperazineethanesulfonic

131 acid, $\mathrm{pH}$ adjusted with $\mathrm{HCl}$ ). To test if the transporters of phosphate or sulfite were involved in

132 selenite uptake, a P or S starvation treatment was conducted in a pretreatment solution before the

133 uptake process. The composition of the pretreatment solution was $15 \mathrm{~g} / \mathrm{L}$ glucose, $3 \mathrm{~g} / \mathrm{L}$ arginine,

$1341.5 \mathrm{~g} / \mathrm{L} \mathrm{K}_{2} \mathrm{HPO}_{4} 0.6 \mathrm{~g} / \mathrm{L} \mathrm{MgSO}_{4} \cdot 7 \mathrm{H}_{2} \mathrm{O}$ for the control treatment. The $\mathrm{pH}$ of the solution was

135 buffered at 6.0 with $2 \mathrm{mM}$ MES. In the $-\mathrm{P}$ or $-\mathrm{S}$ treatment, the $\mathrm{K}_{2} \mathrm{HPO}_{4}$ or $\mathrm{MgSO} 4 \cdot 7 \mathrm{H} 2 \mathrm{O}$ were

136 replaced by the corresponding chloride salts.

137 After shaking cultivation for 12 days, 30 mycelial pellets (5-10 $\mathrm{mm}$ in diameter) were gently

picked up with tweezers, washed with $100 \mathrm{~mL}$ sterile water and $100 \mathrm{~mL}$ uptake solution, and

then transferred into a $250 \mathrm{~mL}$ Erlenmeyer flask containing $100 \mathrm{~mL}$ uptake solution. After an

adaption process of $30 \mathrm{~min}, 1 \mathrm{~mL} 1 \mathrm{mM} \mathrm{Na}_{2} \mathrm{SeO}_{3}$ (sterilized with a $0.2 \mu \mathrm{m}$ filter) was added to

the Erlenmeyer flask to obtain a final selenite concentration of $0.01 \mathrm{mM}$ and start the uptake

process. The flask was then stoppered and shaken at $120 \mathrm{rpm}$ and $25^{\circ} \mathrm{C}$ for $60 \mathrm{~min}$. The uptake

process was stopped by transferring the mycelial pellets to the desorption solution $\left(4^{\circ} \mathrm{C}\right)$. After

desorption for $15 \mathrm{~min}$, the mycelial pellets were sopped up with filter paper and dried at $50^{\circ} \mathrm{C}$.

For the 2,4-DNP (2,4-Dinitrophenol) treatment, $100 \mu \mathrm{L} 0.1 \mathrm{M}$ 2,4-DNP dissolved in ethanol was

added to the uptake solution (a final 2,4-DNP concentration of $0.1 \mathrm{mM}$ ) after the adaption

process. Thirty minutes later, the uptake process was started as stated above. An additional 
148 149 150

control treatment of $0.1 \%(\mathrm{v} / \mathrm{v})$ ethanol was included. For the $-\mathrm{P}$ or $-\mathrm{S}$ treatment, the mycelial pellets were cultivated in the pretreatment solution for $24 \mathrm{~h}$ before transferring into the uptake solution. A control treatment without P or S starvation was included. All the uptake experiments were performed under sterile conditions. Four replicates were conducted for each treatment.

The oven-dried mycelial pellets were grounded in an agate mortar. A subsample of $\sim 0.1 \mathrm{~g}$ was weighted with an analytical balance $( \pm 0.0001 \mathrm{~g})$ and digested with $8 \mathrm{~mL} 68 \%-70 \% \mathrm{HNO}_{3}$ in a microwave oven (CEM Mars 6, CEM, USA). A standard reference material (Full name: CRM Citrus Leaf, GBW 10020; Se concentration: $0.17 \pm 0.03 \mathrm{mg} \mathrm{Se} / \mathrm{kg}$; produced by: Institute of Geophysical and Geochemical Exploration, Chinese Academy of Geological Sciences, China) and blank samples were digested together with the mycelial pellets. The Se concentration in the solution was determined using an inductively coupled plasma-mass spectrometry (ICP-MS, NexION 300X, Perkin Elmer, USA).

\section{Selenite tolerance and reduction experiments}

The cultivation of $F$. velutipes was carried out in the GY solid and liquid media supplemented with $0-5 \mathrm{mM} \mathrm{Na}_{2} \mathrm{SeO}_{3}$. Selenite was added to the culture medium at $50-55^{\circ} \mathrm{C}$ (solid medium) or room temperature (liquid medium) from a stock solution (1 M) after sterilized with a $0.2 \mu \mathrm{m}$ filter.

The tolerance experiment was conducted in solid and liquid media with initial selenite concentrations of $0-5 \mathrm{mM}$. The 9 selenite concentrations tested were $0,0.001,0.01,0.03,0.1,0.3$, 1,3 and $5 \mathrm{mM}$. For solid cultivation, an isolate ( $7 \mathrm{~mm}$ in diameter) was inoculated onto the center of test plate containing $0-5 \mathrm{mM}$ selenite, and incubated at $25^{\circ} \mathrm{C}$ in the dark. The static 
169

170

171

172

174

cultivation was conducted in a $50 \mathrm{~mL}$ flask containing $20 \mathrm{~mL}$ of medium and was inoculated and cultivated in the same way as the solid cultivation. The biomass of the 20-day-old mycelia was determined after oven-dried at $60^{\circ} \mathrm{C}$. The shaking cultivation was conducted in a $250 \mathrm{~mL}$ flask containing $100 \mathrm{~mL}$ of medium with 2 inocula. After cultivation at $25^{\circ} \mathrm{C}$ and $120 \mathrm{rpm}$ in dark for 13 days, the biomass of the mycelial pellets was determined after oven-dried at $60^{\circ} \mathrm{C}$. The radical growth rate, density, height, pigment secretion and other colony characteristics were recorded daily. Each treatment was performed with 4 replicates for the shaking cultivation and with 5 replicates for the solid and static cultivations. For the other 11 species of mushrooms, the tolerance experiments were conducted in solid cultivation with a selenite concentration of 0.1 $\mathrm{mM}$ in the same way as $F$. velutipes.

For the reduction experiment, the ability of $F$. velutipes to reduce selenite to $\operatorname{Se}(0)$ was determined visually, and the degree of red coloration resulted from $\operatorname{Se}(0)$ formation was used as an indication of reduction (Gharieb et al. 1995; no coloration, pink, pale red, and red represented no, weak, moderate, and strong reduction, respectively). During the tolerance experiment with initial selenite concentrations of $0-5 \mathrm{mM}$, the degree of red coloration of the fungal colonies was recorded daily. In addition, the reduction ability was examined after the full development of the mycelium. For this purpose, $F$. velutipes was first cultivated in selenite-free medium for 14 days until selenite was added to the medium to a final selenite concentration of 0 to $3 \mathrm{mM}$. Each treatment was performed in triplicate. The coloration of the mycelial pellets was observed hourly for the first $10 \mathrm{~h}$ and again at $24 \mathrm{~h}$ and $32 \mathrm{~h}$. All of the tolerance and reduction experiments were conducted under sterile conditions. For the other 11 species of mushrooms, the selenite reduction 
190

191

192

193

194

195

196

197

198

199

200

201

202

203

204

205

206

207

208

209

210

was determined visually in solid cultivation $(0.1 \mathrm{mM}$ selenite $)$ and shaking cultivation $(0.3 \mathrm{mM}$ selenite).

For the TEM (transmission electron microscope) studies, the mycelia were picked from the red region of a 20-day-old colony (static cultivation, $0.3 \mathrm{mM}$ selenite) with an inoculating needle and washed 3 times with a $0.01 \mathrm{M}$ PBS solution $\left(\mathrm{NaCl} 8.01 \mathrm{~g} / \mathrm{L}, \mathrm{KCl} 0.20 \mathrm{~g} / \mathrm{L}, \mathrm{Na}_{2} \mathrm{HPO}_{4} \bullet 12 \mathrm{H}_{2} \mathrm{O}\right.$ $\left.3.58 \mathrm{~g} / \mathrm{L}, \mathrm{KH}_{2} \mathrm{PO}_{4} 0.27 \mathrm{~g} / \mathrm{L} ; \mathrm{pH} 7.4\right)$. The mycelia were then suspended in a $1.5 \mathrm{~mL}$ centrifuge tube containing $0.5 \mathrm{~mL}$ of water and scattered in an ultrasonic cleaner. A drop of the obtained mixture was placed onto the copper grids using a rubber head dropper. After drying the sample, a transmission electron microscope (FEI Tecnai G2F20 S-TWIN, USA) working at $200 \mathrm{kv}$ was used for morphology and energy dispersive X-ray spectroscopy (EDX) analysis.

\section{Statistical analysis}

Comparisons among the mycelial biomass in static and shaking cultivations were performed by analysis of variance (ANOVA) followed by Duncan's multiple range test. The mycelial growth after the initial adaption period in the solid cultivation was fitted by a linear regression, and the differences between the slope $(k)$ of the control treatment and the selenite treatment were tested (Table S3). The SAS 9.1 (SAS Institute, Inc., Cary, North Carolina, USA) software package was used for all of the statistical analysis.

\section{Results}

\section{Influences of $\mathbf{p H}$, metabolic inhibitor and nutrient starvation on the selenite uptake}

The rate of selenite uptake was not significantly influenced by the medium $\mathrm{pH}(p>0.05)$, yet its mean values decreased by $14 \%$ as the medium $\mathrm{pH}$ increased from 5.5 to 7.5 (Fig. 1A). The 
211 addition of $0.1 \mathrm{mM}$ 2,4-DNP significantly inhibited the selenite uptake by $11 \%(p<0.05)$ (Fig.

212 1B). The rate of selenite uptake responded similarly to the $\mathrm{P}$ and $\mathrm{S}$ starvation, and decreased by

$21325 \%$ and $26 \%$ after the P and S starvation, respectively $(p<0.05)$ (Fig. 1C).

214 Growth responses of $F$. velutipes to 0-5 $\mathrm{mM}$ selenite

215 The growth rate of $F$. velutipes in the solid cultivation began to be inhibited at $0.1 \mathrm{mM}$ selenite

216 (growth rate decreased by 11\%) (Fig. 2A; Table S3). The inhibition increased with the increasing

217 selenite concentration. The mycelial growth stopped after 5 days in the media containing $1 \mathrm{mM}$

218 selenite (Fig. 2A), and the colony margins became corral-like (Fig. S1). No mycelial growth was

219 observed at the selenite concentrations of $3 \mathrm{mM}$ or higher (Fig. 2A). After different durations of

220

exposures to 3 or $5 \mathrm{mM}$ selenite, the inocula were then re-inoculated to the selenite-free media.

221

Longer lag periods were observed for the selenite stressed inocula, whereas the growth rates after

germination were not severely impaired, except when the inocula were exposed to $5 \mathrm{mM}$ selenite

for 65 days (Fig. 2B; Table S4). In the static cultivation, the inhibition of mycelial growth started

when the selenite concentration reached $0.1 \mathrm{mM}$, and the inhibition intensified as the selenite

concentration increased. No mycelial growth was observed in the media containing selenite

concentrations of $3 \mathrm{mM}$ or higher (Fig. 3C). In the shaking cultivation, the responses of mycelial

growth were similar to those in the static cultivation, except that significant growth inhibition

started at the selenite concentration of $0.3 \mathrm{mM}$ (Fig. 3D).

\section{Reduction of selenite to $\operatorname{Se}(0)$ by $F$. velutipes exposed to 0-5 $\mathrm{mM}$ of selenite}

When F. velutipes was cultivated in the media containing $0-5 \mathrm{mM}$ selenite (Fig. 3A, 3B and 3C),

the reduction intensities, as indicated by the red coloration, increased with increasing selenite 
232

233

234

235

236

237

238

239

240

241

242

243

244

concentrations in the range of $0-0.3 \mathrm{mM}$. Faint reduction appeared at the selenite concentration of $0.03 \mathrm{mM}$ and the reduction intensity increased until $0.3 \mathrm{mM}$. The reduction intensity decreased at the selenite concentration of $1 \mathrm{mM}$. At the selenite concentrations of $3 \mathrm{mM}$ or higher, there was no sign of mycelial growth or selenite reduction. When the mycelial pellets cultivated in the selenite-free media for 14 days were subjected to $0-3 \mathrm{mM}$ selenite, the reduction intensity increased with increasing selenite concentrations (Fig. 3D). In the solid and static cultivation, denser colors were observed in the central part of the colony (around the inocula) compared to the margins (Fig. 3A and 3B; Fig. S2). In the shaking cultivation, denser colors were observed in the large mycelial pellets, especially those developed from the inocula, than in the newly formed small pellets (Fig. 3C and 3D; Fig. S2).

\section{Discussion}

\section{Possible mechanisms of selenite uptake by $F$. velutipes}

The changes in selenite forms induced by the varying $\mathrm{pH}$ did not significantly influence the selenite uptake by F. velutipes (Fig. 1A). From pH 5.5 to $\mathrm{pH}$ 7.5, the proportion of $\mathrm{HSeO}_{3}{ }^{-}$ decreased markedly from $92.6 \%$ to $11.2 \%$, corresponding to the increase of $\mathrm{SeO}_{3}{ }^{2-}$ from $7.3 \%$ to $88.8 \%$ (Table S5; The proportions of different forms were calculated based on the $p K_{a}$ values of selenous acid which determine the degree of the protonation of selenite under different $\mathrm{pH}$ values: $\left.p K_{1}=2.57, p K_{2}=6.60\right)$, whereas the selenite uptake rate only decreased by $14 \%$. The lack of sensitivity of selenite uptake to medium $\mathrm{pH}$ of this range was commonly reported in organisms including algae (Araie et al. 2011; Morlon et al. 2006) and higher plants (Zhang et al. 2006; Zhang et al. 2010; Zhao et al. 2010). Thus, when the $\mathrm{pH}$ of medium is higher than 5, selenite 
253 speciation may not be the key regulator of selenite uptake. However, it was evident that in our 254 and other studies (Araie et al. 2011; McDermott et al. 2010; Zhang et al. 2006; Zhang et al. 2010; 255 Zhao et al. 2010), selenite uptake showed a decreasing trend as medium pH increased from 5 to 8. 256 This may originate from the selenite-proton symport according to the findings of McDermott et 257 al. (2010). Therefore, in F. velutipes, a small portion of the selenite might be taken up through a 258 proton-coupled manner.

259 A minor fraction of the selenite was absorbed metabolically by $F$. velutipes. During our shortterm uptake experiment, 2,4-DNP (the uncoupler of oxidative phosphorylation) inhibited the selenite uptake by $11 \%$ (Fig. 1B). In algae and yeast, the $\Delta \mathrm{pH}$ generated by $\mathrm{H}^{+}$-ATPase has been considered as the driving force of the active selenite transport (Araie et al. 2011; McDermott et al. 2010). In combination with the responses of selenite uptake to medium $\mathrm{pH}$, the active selenite absorption by $F$. velutipes may be associated with the anion-proton symporters. The inhibition rate observed in this study was lower compared with similar studies using 2,4-DNP or CCCP (carbonyl cyanide m-chlorophenyl hydrazone) as metabolic inhibitors at near neutral $\mathrm{pH}$ (inhibition rates ranging from $\sim 25 \%$ to $80 \%$ ) (Gharieb \& Gadd 2004; Li et al. 2008; Zhang et al. 2010). This highlighted the significance of the passive transport in the selenite uptake by $F$. velutipes. Gharieb \& Gadd (2004) observed a fast, metabolism-independent phase in the selenite uptake process of $S$. cerevisiae, and this phase was responsible for the majority of the absorbed

Se. The authors attributed this phase to abiotic adsorption and simple diffusion (Gharieb \& Gadd 2004). Nevertheless, it was unlikely that the selenite uptake in our study proceeded via adsorption or simple diffusion, since the negatively charged cell membrane would repel $\mathrm{HSeO}_{3}{ }^{-}$ 
274 and the hydrophobic core of lipid bilayer was impermeable to selenite (Araie et al. 2011; Morlon

275 et al. 2006; Wells \& Richardson 1985). Moreover, the desorption procedure adopted in this study

276 following the selenite uptake process further precluded the possibility of abiotic adsorption (see

277 Material \& Methods). Therefore, a carrier-facilitated passive transport may be crucial to the

278 selenite uptake by $F$. velutipes.

279 Specific selenite transporters have not been identified, and like other trace anions, selenite may share transporters with the major anions such as phosphate (Lazard et al. 2010; Li et al. 2008; Riedel \& Sanders 1996), sulfite (Zhang et al. 2006) and monocarboxylate (McDermott et al. 2010). We tested the possible roles of phosphate and sulfite transporters in selenite uptake, and assumed that with the up-regulation of phosphate or sulfite transporter after $\mathrm{P}$ or S starvation, the rate of selenite uptake would increase ( $\mathrm{Li}$ et al. 2008). Opposite to our assumption, the uptake rate of selenite decreased significantly by $\sim 25 \%$ after $\mathrm{P}$ and $\mathrm{S}$ starvation (Fig. 1C). One explanation is that two transporters with contrasting selectivity for selenite might exist in $F$. velutipes. In $S$. cerevisiae, which is phylogenetically close to $F$. velutipes, the selenite uptake involves the high and low affinity phosphate transporters at low and high $(1 \mathrm{mM})$ phosphate concentrations, respectively (Lazard et al. 2010). The high affinity transporter strongly selects phosphate over selenite, whereas the low affinity transporter does not discriminate efficiently between the two anions (Lazard et al. 2010). Thus the low affinity transporter can potentially have a higher conductance to selenite than the high affinity transporter (Lazard et al. 2010).

Pinson et al. (2004) observed a close relationship between selenite resistance and the expression of the high affinity phosphate transporter in $S$. cerevisiae. If this transport system applies to $F$. 
295

296

297

298

299

300

301

302

303

304

305

306

307

308

309

310

311

312

313

314

315

velutipes, the transporter with high phosphate affinity would be up-regulated, whereas the one with low affinity would be down-regulated following P starvation. Meanwhile, the low affinity phosphate transporter would be responsible for the selenite uptake in the control treatment with high phosphate concentration $(8.6 \mathrm{mM})$. Because the high affinity transporter discriminate selenite more efficiently, less selenite is absorbed after P starvation than in the control treatment. Similar sulfite transport system might be responsible for the decrease in selenite uptake after $\mathrm{S}$ starvation. Further studies are needed to identify the transporters of selenite in F. velutipes.

\section{Sensitivity of $F$. velutipes to selenite}

An overdose of selenite interferes with the S and P metabolism (Bryant \& Laishley 1988;

Daniels 1996; Tomei et al. 1995; Weissman \& Trelease 1955) and leads to oxidative stress by reacting with intracellular thiols (Papp et al. 2007). In this study, selenite began to be toxic to $F$. velutipes at $0.1 \mathrm{mM}$ (Fig. 2). Based on the colony morphologies, the responses of $F$. velutipes to $0.1 \mathrm{mM}$ selenite were moderate among the commonly cultivated mushrooms (Table S1, S2 and S6). Lin et al. (1997) reported that $0.03 \mathrm{mM}$ and $0.12 \mathrm{mM}$ selenite started to inhibit the growth of F. velutipes in solid and liquid cultivations (PDA media), respectively. The discrepancies with our results in selenite sensitivity may be attributed to the differences in strains and medium components. The corral-like colony margins of $F$. veluptipes when subjected to $1 \mathrm{mM}$ selenite for more than 5 days were also reported when Fusarium sp. was exposed to $5 \mathrm{mM}$ selenite (Gharieb et al. 1995). It remains unclear if this is a common response of fungal mycelia when subjected to toxic amount of selenite. The mycelial growth completely stopped at selenite concentrations of 3 and $5 \mathrm{mM}$ (Fig. 2A, 2C and 2D), but the inocula resuscitated without a 
316 severe decrease in the growth rate after transferred to selenite-free medium (Fig. 2B; Table S4).

317 Thus, a high concentration of selenite induced the dormancy of the inocula without severely

318 damaging their vitality. Based on our results, we recommend a selenite concentration between

$3190.03 \mathrm{mM}$ and $0.1 \mathrm{mM}$ to maintain the balance between Se enrichment and mycelial productivity.

320 This is in agreement with the optimal selenite concentration $(0.06 \mathrm{mM})$ proposed by Ma et al.

321 (2012) for the soluble organic Se accumulation during the fermentation of $F$. velutipes mycelia.

322

323

324

325

326

327

328

329

330

331

332

333

334

335

336

\section{Reduction of selenite to $\operatorname{Se}(0)$ by $F$. velutipes}

The alteration of colony color and the TEM analysis of the red part of the mycelia confirmed the existence of $\operatorname{Se}(0)$ (partly as nanoparticles) when $F$. velutipes was treated with selenite (Fig. 3 and 4). The method of $\operatorname{Se}(0)$ determination in this study (based on colony color; Gharieb et al. 1995) was semiquantitative, because the filamentous nature of the fungal mycelia made it difficult for the use of the spectrophotometric method (Dhanjal \& Cameotra 2010). Further research is needed to improve the accuracy of $\operatorname{Se}(0)$ determination during fungal cultivation. Moreover, the TEM image shows $\operatorname{Se}(0)$ particles outside the hyphae of $F$. velutipes (Fig. 4A). It remains unclear whether there was an extracellular pathway for selenite reduction or the $\operatorname{Se}(0)$ particles inside the hyphae were leaked out during sample preparation. In addition to yeasts (Falcone \& Nickerson 1963; Gharieb et al. 1995; Nickerson \& Falcone 1963) and molds (Brady et al. 1996; Gharieb et al. 1995; Ramadan et al. 1988), some species of mushrooms, including Coriolus versicolor and Lentinula edodes, Ganoderma lucidum, Pleurotus ostreatus and Grifola frondosa (Gharieb et al. 1995; Vetchinkina et al. 2013; Vetchinkina et al. 2016) have been studied for their abilities to transform selenite to $\operatorname{Se}(0)$. In our study, F. velutipes and other 11 
337 species of phylogenetically and ecologically varied mushrooms were tested, and 10 of them

338 showed the reducing ability (Fig. S3). Thus it is conceivable that mushrooms commonly possess

339 this reducing ability.

340 Selenite reduction by fungi to $\operatorname{Se}(0)$ has generally been considered as a detoxification

341 mechanism (Gharieb et al. 1995; Ramadan et al. 1988; Vetchinkina et al. 2013). If the same is

342 true for $F$. velutipes, the reduction intensity would increase as selenite poisoning strengthens. At

343 selenite concentrations below $0.3 \mathrm{mM}$, the reduction intensities increased with the increasing

344 selenite concentration (Fig. 3A, 3B and 3C). However, at selenite concentrations of $1 \mathrm{mM}$ or

345 higher, when the mycelial growth was severely inhibited (Fig. 2), the reduction intensities

346 decreased (Fig. 3A, 3B and 3C). Lortie et al. (1992) also reported that selenite poisoning at high

347 concentrations caused a decrease in the reduction rate of selenite by Pesudomonas stutzeri.

348 Nickerson \& Falcone (1963) proposed that the reduction process operated at the expense of the

349 endogenous metabolism. Selenite acts as a prooxidant at high concentrations which undergoes

350 glutathione-mediated reduction to hydrogen selenide (Izquierdo et al. 2010). The depletion of

351 metabolic products, such as glutathione, might be the reason that the $\operatorname{Se}(0)$ production decreased

352 at high selenite concentrations, and thus we assume that the reduction of selenite to $\operatorname{Se}(0)$ by $F$.

353 velutipes may be metabolism dependent. This idea was tested using mycelial pellets from

354 selenite-free media (14-day-old), as they were supposed to be metabolically active compared

355 with those developed in the media initially containing toxic amounts of selenite. When the

356 mycelia were not constrained by the metabolic activity, the red coloration appeared in $24 \mathrm{~h}$ and

357 the reduction intensity increased with the increasing selenite concentrations in the range of 0-3 
358

359

360

361

362

363

364

365

366

367

368

369

370

371

372

373

374

375

376

377

378

$\mathrm{mM}$ (Fig. 3D). This may explain why the inoculum part of the colony showed faster and denser coloration when subjected to selenite (Fig. 3; Fig. S2). Thus, the reduction of selenite to $\operatorname{Se}(0)$ can be influenced by both the selenite concentration and the metabolism activity of the fungi.

In addition to the red coloration, colonies treated with a toxic amount of selenite often exhibited a garlic smell during cultivation of F. velutipes (Fig. 3C and 3D). Similar phenomena have been reported in the literature studying the responses of fungi to selenite, and the garlic smell was suspected to originate from volatile Se-containing compounds, possibly dimethylselenide (Brady et al. 1996; Gharieb et al. 1995; Schilling et al. 2011). Direct evidence is needed to confirm the speciation of the volatile compounds. This may suggest that the reduction of selenite to $\operatorname{Se}(0)$ was not the only mechanism for $F$. velutipes to cope with the selenite toxicity. The transformation of selenite to volatile Se species which might appear in this study, together with the reduced uptake (Gharieb et al. 1995) and the reduced incorporation of Se into proteins (Ramadan et al. 1988), may also contribute to the tolerance.

\section{Conclusions}

At near neutral $\mathrm{pH}$, selenite uptake was slightly affected by selenite species and metabolic inhibitor. A carrier-mediated passive transport might be crucial to the selenite absorption by $F$. velutipes. At selenite concentrations of $0.1 \mathrm{mM}$ or higher, the absorbed selenite led to growth inhibition of $F$. velutipes. As a detoxification mechanism, a portion of the selenite was transformed to $\mathrm{Se}(0)$ including $\mathrm{Se}(0)$ nanoparticles in a metabolic dependent way. These results provided some basic information for the cultivation of the selenized $F$. velutipes and highlighted the opportunity of using mushrooms for the production of $\operatorname{Se}(0)$ nanoparticles. 


\section{References}

380

Araie H, Sakamoto K, Suzuki I, and Shiraiwa Y. 2011. Characterization of the selenite uptake mechanism in the coccolithophore Emiliania huxleyi (Haptophyta). Plant and Cell Physiology 52: 2014-1210.

Brady JM, Tobin JM, and Gadd GM. 1996. Volatilization of selenite in aqueous medium by a Penicillium species. Mycological Research 100:955-961.

Bryant RD, and Laishley EJ. 1988. Evidence for two transporters of sulfur and selenium oxyanions in Clostridium pasteurianum. Canadian Journal of Microbiology 34:700-703.

Cheung L, Cheung PC, and Ooi VE. 2003. Antioxidant activity and total phenolics of edible mushroom extracts. Food Chemistry 81:249-255.

Chun JY, Nadiminty N, Lee SO, Onate SA, Lou W, and Gao AC. 2006. Mechanisms of selenium down-regulation of androgen receptor signaling in prostate cancer. Molecular Cancer Therapeutics 5:913-918.

Combs GF. 2001. Selenium in global food systems. British Journal of Nutrition 85:517-547.

Daniels LA. 1996. Selenium metabolism and bioavailability. Biological Trace Element Research 54:185-199.

Dhanjal S, and Cameotra SS. 2010. Aerobic biogenesis of selenium nanospheres by Bacillus cereus isolated from coalmine soil. Microbial Cell Factories 9: 52.

Dong JZ, Lei C, Ai XR, and Wang Y. 2012. Selenium enrichment on Cordyceps militaris Link and analysis on its main active components. Applied Biochemistry and Biotechnology 166:1215-1224.

Egressy-Molnár O, Ouerdane L, Győrfi J, and Dernovics M. 2016. Analogy in selenium enrichment and selenium speciation between selenized yeast Saccharomyces cerevisiae and Hericium erinaceus (lion's mane mushroom). LWT - Food Science and Technology 68:306-312.

Falandysz J. 2008. Selenium in edible mushrooms. Journal of Environmental Science and Health, Part C: Environmental Carcinogenesis and Ecotoxicology Reviews 26:256-299.

Falcone G, and Nickerson WJ. 1963. Reduction of selenite by intact yeast cells and cell-free preparations. Journal of Bacteriology 85:754-762.

Gharieb M, Wilkinson S, and Gadd G. 1995. Reduction of selenium oxyanions by unicellular, polymorphic and filamentous fungi: cellular location of reduced selenium and implications for tolerance. Journal of Industrial Microbiology 14:300-311. 
406 Gharieb MM, and Gadd GM. 2004. The kinetics of ${ }^{75}[\mathrm{Se}]$-selenite uptake by Saccharomyces cerevisiae and the

407 vacuolization response to high concentrations. Mycological Research 108:1415-1422.

Izquierdo A, Casas C, and Herrero E. 2010. Selenite-induced cell death in Saccharomyces cerevisiae: protective role of glutaredoxins. Microbiology 156:2608-2620.

Jing P, Zhao S-J, Lu M-M, Cai Z, Pang J, and Song L-H. 2014. Multiple-Fingerprint Analysis for Investigating Quality Control of Flammulina velutipes Fruiting Body Polysaccharides. Journal of Agricultural and Food Chemistry 62:12128-12133.

Lazard M, Blanquet S, Fisicaro P, Labarraque G, and Plateau P. 2010. Uptake of selenite by Saccharomyces cerevisiae involves the high and low affinity orthophosphate transporters. Journal of Biological Chemistry 285:32029-32037.

Levine VE. 1925. The reducing properties of microorganisms with special reference to selenium compounds. Journal of Bacteriology 10:217.

Li HF, McGrath SP, and Zhao FJ. 2008. Selenium uptake, translocation and speciation in wheat supplied with selenate or selenite. New Phytologist 178:92-102.

Lin L, Xie BF, Shi QG, and Zhang WH. 1997. Submerged fermentation of Se-accumulating Flammulina velutipes and its characters. Edible Fungi of China 16:36-38. in Chinese with English abstract.

Lortie L, Gould W, Rajan S, McCready R, and Cheng K-J. 1992. Reduction of selenate and selenite to elemental selenium by a Pseudomonas stutzeri isolate. Applied and Environmental Microbiology 58:4042-4044.

Lovley DR. 1993. Dissimilatory metal reduction. Annual Reviews in Microbiology 47:263-290.

Mániková D, Vlasáková D, Loduhová J, Letavayová L, Vigašová D, Krascsenitsová E, Vlčková V, Brozmanová J, and Chovanec M. 2010. Investigations on the role of base excision repair and non-homologous end-joining pathways in sodium selenite-induced toxicity and mutagenicity in Saccharomyces cerevisiae. Mutagenesis $25: 155-162$.

Ma Y, Xiang F, Xiang J, and Yu L. 2012. Optimization of Soluble Organic Selenium Accumulation during Fermentation of Flammulina velutipes Mycelia. Zeitschrift für Naturforschung C 67:594-602.

Maseko T, Callahan DL, Dunshea FR, Doronila A, Kolev SD, and Ng K. 2013. Chemical characterisation and speciation of organic selenium in cultivated selenium-enriched Agaricus bisporus. Food Chemistry 
141:3681-3687.

434

McDermott JR, Rosen BP, and Liu Z. 2010. Jen1p: a high affinity selenite transporter in yeast. Molecular Biology of the Cell 21:3934-3941.

Morlon H, Fortin C, Adam C, and Garnier-Laplace J. 2006. Selenite transport and its inhibition in the unicellular green alga Chlamydomonas reinhardtii. Environmental Toxicology and Chemistry 25:1408-1417.

Nickerson WJ, and Falcone G. 1963. Enzymatic reduction of selenite. Journal of Bacteriology 85:763-771.

Niedzielski P, Mleczek M, Siwulski M, Rzymski P, Gąsecka M, and Kozak L. 2015. Supplementation of cultivated mushroom species with selenium: bioaccumulation and speciation study. European Food Research and Technology:1-8.

Nunes RGFL, Luz JMRd, Freitas RdB, Higuchi A, Kasuya MCM, and Vanetti MCD. 2012. Selenium Bioaccumulation in Shiitake Mushrooms: A Nutritional Alternative Source of this Element. Journal of Food Science 77:983-986.

Obata T, Araie H, and Shiraiwa Y. 2004. Bioconcentration mechanism of selenium by a coccolithophorid, Emiliania huxleyi. Plant and Cell Physiology 45:1434-1441.

Oremland RS, Herbel MJ, Blum JS, Langley S, Beveridge TJ, Ajayan PM, Sutto T, Ellis AV, and Curran S. 2004. Structural and spectral features of selenium nanospheres produced by Se-respiring bacteria. Applied and Environmental Microbiology 70:52-60.

Papp LV, Lu J, Holmgren A, and Khanna KK. 2007. From selenium to selenoproteins: synthesis, identity, and their role in human health. Antioxidants \& redox signaling 9:775-806.

Pinson B, Merle M, Franconi J-M, and Daignan-Fornier B. 2004. Low affinity orthophosphate carriers regulate PHO gene expression independently of internal orthophosphate concentration in Saccharomyces cerevisiae. Journal of Biological Chemistry 279:35273-35280.

Ramadan SE, Razak A, Yousseff Y, and Sedky N. 1988. Selenium metabolism in a strain of Fusarium. Biological Trace Element Research 18:161-170.

Riedel GF, and Sanders JG. 1996. The influence of pH and media composition on the uptake of inorganic selenium by Chlamydomonas reinhardtii. Environmental Toxicology and Chemistry 15:1577-1583.

Rzymski P, Mleczek M, Niedzielski P, Siwulski M, and Gąsecka M. 2016. Potential of cultivated Ganoderma 
460

461

462

463

464

465

466

467

468

469

470

471

472

473

474

475

476

477

478

479

480

481

482

483

484

485

486

lucidum mushrooms for the production of supplements enriched with essential elements. Journal of Food Science 81:C587-C592.

Schilling K, Johnson TM, and Wilcke W. 2011. Isotope Fractionation of selenium during fungal biomethylation by Alternaria alternata. Environmental Science \& Technology 45:2670-2676.

Stolz JF, and Oremland RS. 1999. Bacterial respiration of arsenic and selenium. FEMS Microbiology Reviews 23:615-627.

Suhajda A, Hegoczki J, Janzso B, Pais I, and Vereczkey G. 2000. Preparation of selenium yeasts I. Preparation of selenium-enriched Saccharomyces cerevisiae. Journal of Trace Elements in Medicine and Biology 14:43-47.

Tomei FA, Barton LL, Lemanski CL, Zocco TG, Fink NH, and Sillerud LO. 1995. Transformation of selenate and selenite to elemental selenium by Desulfovibrio desulfuricans. Journal of Industrial Microbiology 14:329336.

Turło J, Gutkowska B, and Herold F. 2010a. Effect of selenium enrichment on antioxidant activities and chemical composition of Lentinula edodes (Berk.) Pegl. mycelial extracts. Food and Chemical Toxicology 48:10851091.

Turło J, Gutkowska B, Herold F, Gajzlerska W, Dawidowski M, Dorociak A, and Zobel A. 2011. Biological Availability and Preliminary Selenium Speciation in Selenium-Enriched Mycelium of Lentinula edodes (Berk.). Food Biotechnology 25:16-29.

Turło J, Gutkowska B, Herold F, Klimaszewska M, and Suchocki P. 2010b. Optimization of selenium-enriched mycelium of Lentinula edodes (Berk.) pegler as a food supplement. Food Biotechnology 24:180-196.

Vetchinkina E, Loshchinina E, Kursky V, and Nikitina V. 2013. Reduction of organic and inorganic selenium compounds by the edible medicinal basidiomycete Lentinula edodes and the accumulation of elemental selenium nanoparticles in its mycelium. Journal of Microbiology 51:829-835.

Vetchinkina E, Loshchinina E, Kurskyi V, and Nikitina V. 2016. Biological synthesis of selenium and germanium nanoparticles by xylotrophic basidiomycetes. Applied Biochemistry and Microbiology 52:87-97.

Weissman GS, and Trelease SF. 1955. Influence of sulfur on the toxicity of selenium to Aspergillus. American Journal of Botany:489-495. 
487 Wells J, and Richardson D. 1985. Anion accumulation by the moss Hylocomium splendens: uptake and competition

488

489

490

491

492

493

494

495

496

497 studies involving arsenate, selenate, selenite, phosphate, sulphate and sulphite. New Phytologist 101:571583.

Yan H, and Chang H. 2012. Antioxidant and Antitumor Activities of Selenium-and Zinc-Enriched Oyster Mushroom in Mice. Biological Trace Element Research 150:236-241.

Yang W, Yu J, Pei F, Mariga AM, Ma N, Fang Y, and Hu Q. 2016. Effect of hot air drying on volatile compounds of Flammulina velutipes detected by HS-SPME-GC-MS and electronic nose. Food Chemistry 196:860-866.

Zhang L, Li D, and Gao P. 2012. Expulsion of selenium/protein nanoparticles through vesicle-like structures by Saccharomyces cerevisiae under microaerophilic environment. World Journal of Microbiology and Biotechnology 28:3381-3386.

Zhang L, Shi W, and Wang X. 2006. Difference in selenite absorption between high-and low-selenium rice cultivars and its mechanism. Plant and Soil 282:183-193.

Zhang L, Yu F, Shi W, Li Y, and Miao Y. 2010. Physiological characteristics of selenite uptake by maize roots in response to different pH levels. Journal of Plant Nutrition and Soil Science 173:417-422.

Zhao XQ, Mitani N, Yamaji N, Shen RF, and Ma JF. 2010. Involvement of silicon influx transporter OsNIP2; 1 in selenite uptake in rice. Plant Physiology 153:1871-1877.

Zhu YG, Pilon-Smits EA, Zhao FJ, Williams PN, and Meharg AA. 2009. Selenium in higher plants: understanding mechanisms for biofortification and phytoremediation. Trends in Plant Science 14:436-442. 
1

Influences of $\mathrm{pH}(\mathrm{A})$, metabolic inhibitor (B) and P or S starvation (C) on the selenite uptake by $F$. velutipes.

Different letters indicate statistical difference at the 0.05 level.
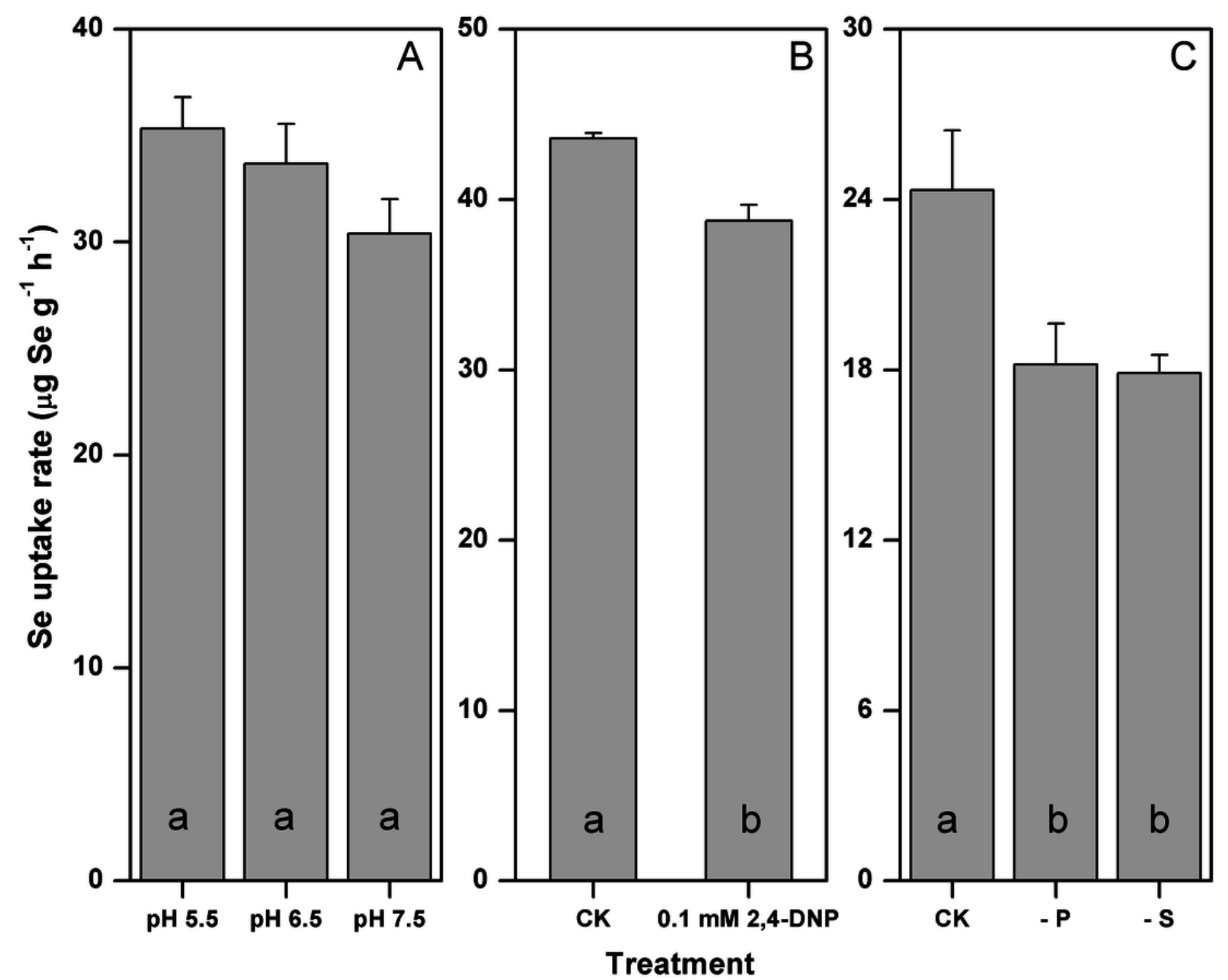
2

Growth responses of $F$. velutipes to 0-5 mM selenite in solid cultivation (A and B), static cultivation (C) and shaking cultivation (D).

In panel (D), the inocula were exposed to $3 \mathrm{mM}$ or $5 \mathrm{mM}$ selenite in solid cultivation for $20-65$ days and then were inoculated onto the selenite-free media. Different letters indicate statistical difference at the 0.05 level.
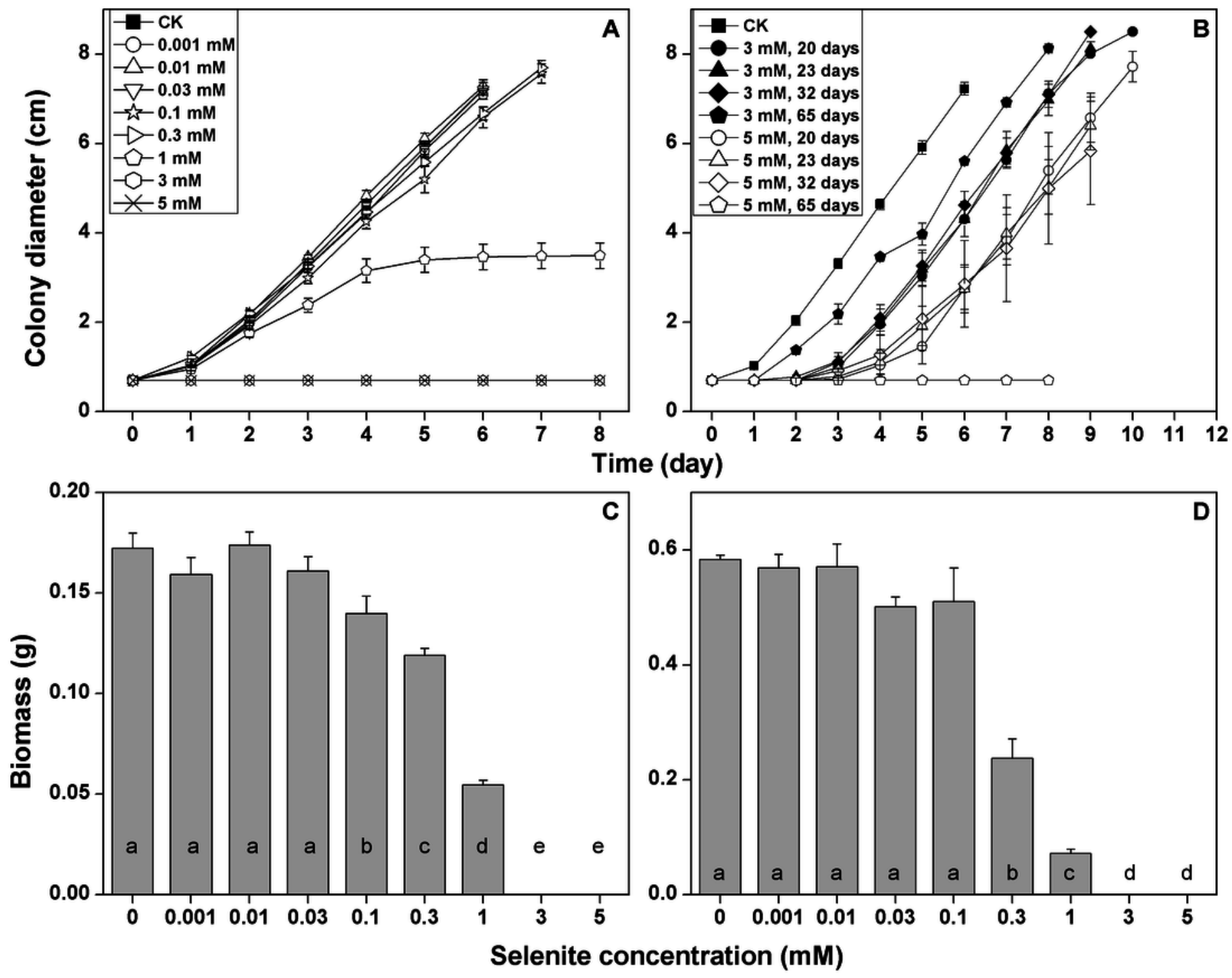
3

The reduction intensities of $F$. velutipes supplied with 0-5 mM selenite (indicated by the red colorations of the colonies).

The isolates from the stock colonies were inoculated into the media containing 0-5 mM selenite and were subjected to solid cultivation (A), static cultivation (B) and shaking cultivation (C). In another experiment (D), after shaking cultivation in selenite-free media for 14 days, the resulting mycelial pellets were treated with 0-3 mM selenite. The first pink circle indicates the beginning of the red coloration. The small circle inside represents the central part of the colony ( $A$ and $B$ ) or the large mycelial pellet ( $C$ and $D)$, and the ring outside represents the margin of the colony ( $A$ and $B$ ) or the small mycelial pellet ( $C$ and $D$ ). The plus/minus signs on the right side of circles in panels (C) and (D) represent the intensities of garlic smell after selenite treatment (-: none; + : low; ++ : moderate; +++ : strong). 

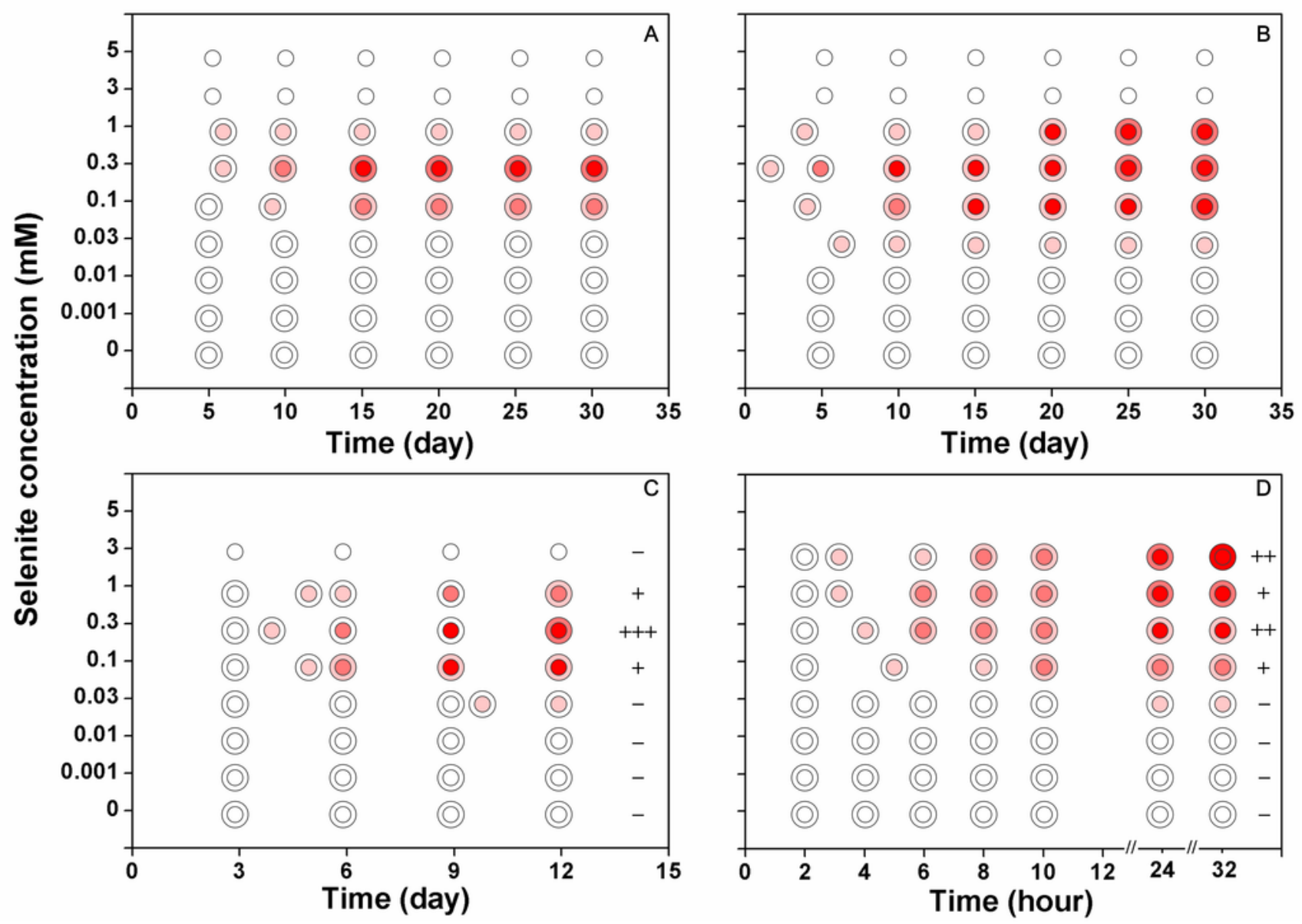
4

TEM (A) and EDX (B) images of the hyphae of $F$. velutipes after selenite treatment.

The mycelia were picked up from the red region of the 20-day-old colony in the static cultivation supplied with $0.3 \mathrm{mM}$ selenite.
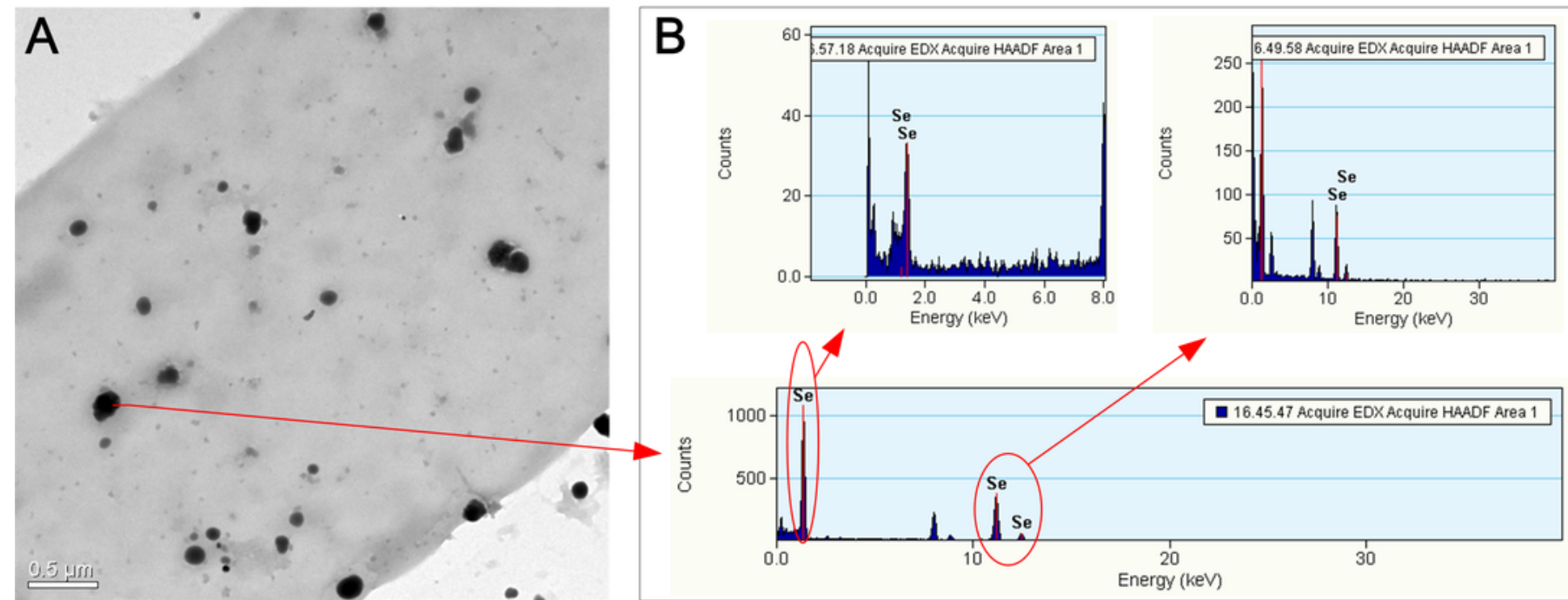